International Journal of Biomedicine | June 2019 - Volume 9, Issue Suppl_1: Abstracts From the Second Russian International Conference "Cryo-electron microscopy 2019: achievements and prospects"

POSTER ABSTRACT PRESENTATIONS

SESSION TITLE: STRUCTURE OF MEMBRANE PROTEINS

DOI: 10.21103/IJBM.9.Suppl_1.P3

\title{
Abstract P-3: Nanoparticles with Ion Channels: Solubilization and Purification of Membrane Proteins
}

\author{
Julia Kacher, Grigory Glukhov, Olga Sokolova \\ Lomonosov Moscow State University, Moscow, Russia
}

Background: Now there are several approaches to isolate membrane proteins without detergents. Such approaches are promising as they guarantee the preservation of the lipid environment of the target proteins. One of such novel methods is based on the usage of a copolymer of styrene and maleic acid (SMA). Particles with small membrane fragments containing transmembrane proteins bounded by a polymer are called nanodisks (SMALPs).

Methods: We chose HEAG2 with affine 1d4 tag at the C-terminus as an object of interest. Channels were expressed in COS-1 eukaryotic cell line (African green monkey kidney fibroblast-like cell line). The potassium channels were integrated into SMALPs during the solubilization and then purified via affinity chromatography. The embedding of the channel into the particles with SMA was confirmed by immunoblotting. Dynamic light scattering was used to confirm the formation of SMALPs with channel protein. Obtained samples were subjected to negative stain electron microscopy.

Results: We have succeeded to extract voltage-gated potassium channels from the membrane fractions directly. The hydrodynamic diameter of the formed particles was $15.5 \mathrm{~nm}$. The analysis of EM images also confirmed the formation of nanoparticles.

Conclusion: The technique for solubilizing and purifying ion channels from eukaryotic cells using SMA allows to make three-dimensional reconstruction of the particles.

Key Words: Ion channels $\bullet$ detergent-free solubilization methods $\bullet$ membrane proteins

Sources of Funding: This work was supported by the grant of the Russian Science Foundation for young scientists (18-74- 00087).Electron microscopy was performed on the basis of the CCP of the Faculty of Biology MSU "Electron microscopy in the science of life" using the 3D-EMC.

International Journal of Biomedicine. 2019;9 Suppl 1: S18. doi: 10.21103/IJBM.9.Suppl_1.P3

C2019 International Medical Research and Development Corporation 